\title{
The impact of rituximab prophylaxis on hepatocellular carcinoma recurrence after living donor liver transplantation
}

\author{
Naoko Kamo, Shintaro Yagi \\ Division of Hepato-Biliary-Pancreatic and Transplant Surgery, Department of Surgery, Graduate School of Medicine, Kyoto \\ University, Kyoto 606-8507, Japan.
}

Correspondence to: Naoko Kamo, MD, PhD, Division of Hepato-Biliary-Pancreatic and Transplant Surgery, Department of Surgery, Graduate School of Medicine, Kyoto University, Kyoto 606-8507, Japan. E-mail: naoko@kuhp.kyoto-u.ac.jp

How to cite this article: Kamo N, Yagi S. The impact of rituximab prophylaxis on hepatocellular carcinoma recurrence after living donor liver transplantation. Hepatoma Res 2021;7:28. https://dx.doi.org/10.20517/2394-5079.2020.147

Received: 14 Nov 2020 First Decision: 28 Dec 2020 Revised: 8 Jan 2021 Accepted: 8 Feb 2021 Available online: 9 Apr 2021

Academic Editors: Nobuhisa Akamatsu, James Fung Copy Editor: Xi-Jun Chen Production Editor: Xi-Jun Chen

\begin{abstract}
Aim: Rituximab is administered for ABO blood type incompatibility or donor-specific anti-HLA (human leukocyte antigen) antibody-positive liver transplantation (LT). However, the impact of rituximab administration on hepatocellular carcinoma (HCC) recurrence over a long term period remains unclear. The present study aimed to retrospectively investigate the impact of rituximab-based prophylaxis on $\mathrm{HCC}$ recurrence after living donor LT (LDLT).

Methods: A total of 117 patients who had undergone LDLT for HCC at Kyoto University between February 2006 and October 2018 were retrospectively enrolled for this study. Overall survival (OS) and the recurrence rate (RR) for HCC after LDLT were examined in patients who received rituximab (rituximab group: $n=31$ ) vs. those who did not (control group: $n=86$ ). Additional analyses were conducted as per the Milan criteria, the University of California San Francisco extended criteria (single tumor $\leq 6.5 \mathrm{~cm}$, or $\leq 3$ nodules with the largest tumor $\leq 4.5 \mathrm{~cm}$, and total tumor diameter $\leq 8 \mathrm{~cm}$ ), and the Kyoto criteria (KC) [maximum size $\leq 5 \mathrm{~cm}$, number $\leq 10$, des-gammacarboxy prothrombin $(D C P) \leq 400]$. Moreover, we analyzed risk factors associated with $\mathrm{HCC}$ recurrence with a focus on pretransplant factors.
\end{abstract}

Results: The one-, three-, and five-year (1/3/5-y) OS and RR for all patients were $89 \% / 81 \% / 79 \%$ and $5 \% / 9 \% / 11 \%$, respectively. The $1 / 3 / 5-y$ OS and $1 / 3 / 5-y$ RR in the rituximab group vs. the control group were $87 \% / 77 \% / 69 \%$ and $4 \% / 4 \% / 8 \%$ vs. $89 \% / 82 \% / 82 \%$ and $5 \% / 11 \% / 12 \%$, respectively $(P=0.11$ and $P=0.55$, respectively). In the subgroup analysis stratified by the selection criteria, the RR was comparable between groups. The number of patients with non-recurrence-related death tended to be higher in the rituximab group than the 
control group. Multivariate analysis identified maximum tumor size $(P=0.003)$ and preoperative treatment $(P=$ 0.024) as independent risk factors for HCC recurrence.

Conclusion: Rituximab administration does not seem to affect HCC recurrence after LDLT.

Keywords: Rituximab, ABO incompatible, hepatocellular carcinoma, recurrence, liver transplantation

\section{INTRODUCTION}

Hepatocellular carcinoma (HCC) is the most common type of primary liver cancer in adults, and it has become the sixth most diagnosed cancer and the fourth leading cause of death worldwide ${ }^{[1]}$. Liver transplantation (LT) is a radical treatment for end-stage liver disease. Notably, for HCC, LT is the ultimate method that not only treats the tumor, but also the underlying original liver disease. However, the shortage of deceased donor livers is an extremely serious problem in Eastern countries. Therefore, living donor (LD) LT has evolved dramatically in the last 3 decades ${ }^{[2,3]}$.

ABO blood-type incompatible (ABO-I) transplantation could be a feasible alternative for LDLT in which a patient receives an organ from a close relative donor. Moreover, given the current situation in Japan, where deceased donor LT is rare, donor-specific anti-HLA (human leukocyte antigen) antibody (DSA)-positive patients who undergo preoperative desensitization therapy similar to ABO-I LT could also be candidates for LDLT $^{[4]}$. However, LDLT for these candidates is often associated with the risk of antibody-mediated rejection (AMR), such as hepatic necrosis or intrahepatic biliary complications, by anti-donor $\mathrm{ABO}$ antibodies or $\mathrm{DSA}^{[5,6]}$. The initial risk of postoperative complications has been ameliorated considerably by applying various approaches ${ }^{[5]}$. Lately, following the advent of preoperative desensitization by rituximab administration and plasma exchange (PE), the outcome of ABO-I LDLT has improved remarkably ${ }^{[7]}$.

Effects associated with rituximab administration may include susceptibility to infection or potential involvement of the immune system that can affect tumor recurrence. There have been some reports of outcomes related to HCC recurrence in patients with ABO-I transplantation in the rituximab era ${ }^{[8-10]}$, but the long-term prognosis following rituximab-based prophylaxis has not been well elucidated.

The present study aimed to analyze the impact of rituximab-based prophylaxis on outcomes in patients with HCC after LDLT, focusing on the Milan criteria and our expanded criteria.

\section{METHODS}

\section{Patients}

A total of 117 consecutive patients who underwent adult LDLT for HCC at the Kyoto University between February 2006 and October 2018 were enrolled. The patients were preoperatively diagnosed with HCC on imaging and those with a re-transplantation were excluded. This study was approved by the ethics committee of Kyoto University (approval no. R1473-3) and was conducted under the Declaration of Helsinki (2000).

\section{Surgical procedures and perioperative management}

Basic selection criteria and the operative techniques for both the donor and recipient, and perioperative management have been described in detail elsewhere ${ }^{[1,12]}$. Three-dimensional reconstructed images of the hepatic vascular anatomy produced using a software package based on reconstructed, multi-detector row, computed tomography (CT) of the liver were used to estimate the graft and remnant liver volumes in donors preoperatively. Treatment for hepatitis $\mathrm{C}$ virus with interferon-containing regimens have been 
administered previously to prevent the progression of hepatitis C after LT. Since 2015, interferon-free therapies with direct-acting antivirals have also been introduced ${ }^{[13]}$. Moreover, combination therapy with lamivudine and hepatitis B immunoglobulin (HBIG) has been administered in patients with hepatitis B virus to prevent recurrence after LT. As such, from December 2006, our team has changed the protocol for prophylaxis to entecavir and $\mathrm{HBIG}^{[14]}$.

\section{Immunosuppressants}

The immunosuppression protocol that consisted of tacrolimus (TAC) and low-dose steroid was used until January $2011^{[15,16]}$. After February 2011, a steroid-free protocol with mycophenolate mofetil (MMF) was adopted, as described elsewhere, except for patients with ABO-I transplants or steroid administration before $\mathrm{LT}^{[17]}$. In the case of ABO-I transplantation, recipients received a single intravenous dose of rituximab 2-3 weeks before LDLT for desensitization. Until 2016, the dose of rituximab was $300 \mathrm{mg}$, but it was changed to either $375 \mathrm{mg} / \mathrm{m}^{2}$ or $500 \mathrm{mg}$ according to results published by Egawa et al. ${ }^{[18]}$. If the decrease of the antibody titer or B cell number was insufficient, an additional dose was administered. In the present study, TAC and MMF were started 7 days before LDLT, starting from $1.0 \mathrm{mg} /$ day and $500 \mathrm{mg} /$ day, respectively [Figure 1C]. Before the transplant, 2 or 3 sessions of PE were also performed. Thereafter, postoperative triple immunosuppression was achieved with TAC, MMF, and methylprednisolone; methylprednisolone was given orally, enterally, or intravenously instead of by arterial infusion [by June 2009; Figure 1A] or portal infusion [by July 2013; Figure 1B]. For DSA-strongly positive transplantation, a series of desensitizations were performed following the ABO-I transplant. In addition to the usual postoperative administration of TAC and MMF, methylprednisolone at a dose of $125 \mathrm{mg}$ was administered for 1 week from the day after the operation. The steroid dose was then gradually tapered. Moreover, 2 patients in the present study also received a mammalian target of rapamycin inhibitor as an immunosuppressant.

\section{Assessment of hepatocellular carcinoma}

In the month before LDLT, the HCC was assessed by contrast-enhanced CT. If patients had preoperative treatments, CT was performed at a 3-month interval. A radiologist confirmed tumor size, tumor number, and the presence of macrovascular invasion or distant metastasis. To verify the presence of HCC preoperatively, confirmation of the presence of HCC nodules by radiologists or pathological evidence of HCC on the explanted liver was needed. The following patients were excluded: those with retransplantation, nodules with complete necrosis, and incidental HCC found only on pathological diagnosis postoperatively.

\section{Selection criteria}

There was no restriction of size or tumor number for LDLT for HCC until December 2006. After January 2007, the Kyoto criteria (KC), which included the following three significant independent risk factors for recurrence, were applied: tumor number $\leq 10$, maximal diameter of each tumor $\leq 5 \mathrm{~cm}$, and DCP concentration $\leq 400 \mathrm{mAU} / \mathrm{mL}^{[19]} .2$ patients who were on warfarin were excluded from the $\mathrm{KC}$ because showed an abnormally high DCP concentration.

\section{Analysis}

The following factors were compared between the rituximab administration group and the control group: age, sex, etiology, alpha-fetoprotein (AFP), DCP, model for end-stage liver disease (MELD) score, ChildPugh classification, graft type, graft-to-recipient body weight ratio (GRWR), operation time, blood loss, splenectomy, tumor radius, tumor number, the pathological classification of each patient, ABO incompatibility, donor-specific anti-HLA antibody-strong positive, AMR, acute T-cell mediated rejection, and recurrence. Overall survival (OS) and recurrence rates (RR) were analyzed after LT in all patients with or without rituximab administration. Next, all recipients were assigned to two subgroups according to 
A

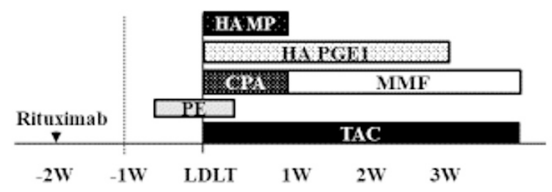

B

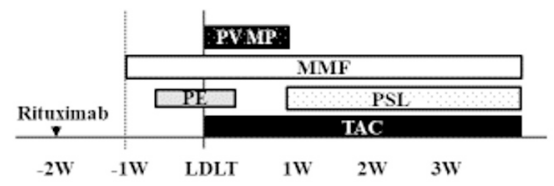

C

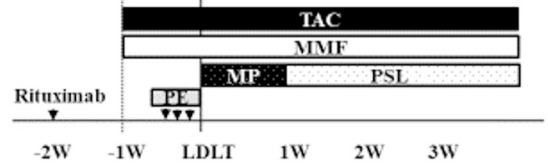

Figure 1. Standard immunosuppressant protocol for ABO-I (A) from April 2006 to June 2009, (B) from July 2009 to July 2013, and (C) from August 2013. CPA: Cyclophosphamide; HA: hepatic artery; MMF: mycophenolate mofetil; MP: methylprednisolone; PE: plasma exchange; PGE1: prostaglandin E1; PV: portal vein; TAC: tacrolimus; PSL: prednisolone.

whether they were within or beyond the Milan, University of California San Francisco (UCSF), or Kyoto criteria, and similarly analyzed. Factors independently associated with HCC recurrence after LT were also analyzed.

\section{Statistical analysis}

Continuous variables are expressed as medians with ranges and categorical variables as numbers. Patient characteristics were compared between the 2 groups using Fisher's exact test or the $c^{2}$ test for categorical variables and the Mann-Whitney $U$ test for continuous variables. Cumulative OS and RR were analyzed by the Kaplan-Meier method and the log-rank test. Any variable identified as significant $(P<0.05)$ in univariate analyses was subject to multivariate Cox proportional-hazard modeling. A $P$ value $<0.05$ was considered significant. All data were calculated using JMP pro version 14 software (SAS Institute, Cary, $\mathrm{NC})$.

\section{RESULTS}

Clinicopathological and perioperative characteristics of the patients are shown in Table 1. The median age was 59 (29-69) years, and 81 patients were male (69\%). The median AFP and median DCP were 29.6 (1.3$28074) \mathrm{ng} / \mathrm{mL}$ and 47 (6-12523) $\mathrm{mAU} / \mathrm{mL}$, respectively. Seventy-five cases (64\%) had a history of preoperative treatment [Figure 2]. Fifty-six cases received transcatheter arterial chemoembolization (TACE), 49 had radio-frequency ablation (RFA), 11 cases had a hepatic resection (HR), and 5 cases received all treatments. The median follow-up period was 86 (1-154) months. One-, three-, and five-year OS rates were $89 \%, 81 \%$, and $79 \%$, respectively [Figure 3A]. One-, three-, and five-year RRs were $5 \%, 9 \%$, and $11 \%$, respectively [Figure $3 \mathrm{~B}]$.

Table 2 shows the clinicopathological and perioperative characteristics of patients with (rituximab group) and without (control group) rituximab administration. Rituximab was administered to 31 patients, including $30 \mathrm{ABO}-\mathrm{I}$ and $3 \mathrm{DSA}$-strongly positive cases. 2 of 3 patients who were DSA-positive were also ABO-I cases. There was a significant difference in tumor numbers between the 2 groups, though the median number was the same. Operation time was significantly longer in the rituximab group than in the control group $(P<0.01)$. As for the pathological classification of tumors, the moderately differentiated type was 
Table 1. Clinico-pathological and perioperative characteristics of patients

\begin{tabular}{|c|c|}
\hline Characteristics & $n=117$ \\
\hline Age (years) & $59(29-69)$ \\
\hline Sex (male/female) & $81(69 \%) / 36$ \\
\hline \multicolumn{2}{|l|}{ Etiology } \\
\hline Viral hepatitis C & $66(56 \%)$ \\
\hline Viral hepatitis B & $29(25 \%)$ \\
\hline Viral hepatitis C/B & $3(3 \%)$ \\
\hline Others & $19(16 \%)$ \\
\hline $\operatorname{AFP}(n g / m L)$ & $29.6(1.3-28074)$ \\
\hline $\mathrm{DCP}(\mathrm{mAU} / \mathrm{mL})$ & $47(6-12523)$ \\
\hline Child Pugh (A/B/C) & $13(11 \%) / 45(39 \%) / 59(50 \%)$ \\
\hline MELD & $15(6-41)$ \\
\hline Maximum tumor size $(\mathrm{cm})$ & $2.2(0.7-8.8)$ \\
\hline Number of tumors & $2(1-186)$ \\
\hline Graft type (rt graft/others) & $83(71 \%) / 34$ \\
\hline GRWR & $0.9(0.56-1.54)$ \\
\hline Operation time (min) & $797(557-1288)$ \\
\hline Blood loss (mL) & $5830(920-30418)$ \\
\hline Classification (well/mod/poor) & $14(12 \%) / 83(71 \%) / 20(17 \%)$ \\
\hline Microvascular invasion & $27(23 \%)$ \\
\hline Preoperative treatment & $75(64 \%)$ \\
\hline Time to transplant (month) & $14(0-120)$ \\
\hline \multicolumn{2}{|l|}{$A B O$ incompatibility } \\
\hline Identical, compatible/incompatible & $87(74 \%) / 30$ \\
\hline \multicolumn{2}{|l|}{ Donor-specific antibody-strong positive } \\
\hline Identical, compatible/incompatible & $1(1 \%) / 2(2 \%)$ \\
\hline Milan criteria (met/exceeding) & $83(71 \%) / 34$ \\
\hline UCSF criteria (met/exceeding) & $89(76 \%) / 28$ \\
\hline Kyoto criteria (met/exceeding) & $94(82 \%) / 21$ \\
\hline
\end{tabular}

AFP: Alpha-fetoprotein; DCP: des-gamma-carboxy prothrombin; GRWR: graft/recipient body weight ratio; MELD: model for end-stage liver disease; UCSF: University of California San Francisco.

significantly more common in the rituximab group than in the control group $(P=0.02)$. The incidence of AMR was significantly higher in the rituximab group than in the control group $(P=0.02) .2$ patients in the rituximab group had a recurrence 10 and 38 months after LT, respectively. On the other hand, 10 patients in the control group had a recurrence. The median period of recurrence was 18 months (range; 1-122 months). 3 of 10 patients in the control group were still alive.

One-, three-, and five-year OS rates in the groups with and without rituximab administration were $87 \%$, $77 \%$, and $69 \%$ vs. $89 \%, 82 \%$, and $82 \%$, respectively; there were no significant differences between the two groups $[P=0.11$; Figure $4 \mathrm{~A}]$. The one-, three-, and five-year RRs in the groups with and without rituximab administration were $4 \%, 4 \%$, and $8 \% v$ s. $5 \%, 11 \%$, and $12 \%$, respectively; there were no significant differences between the groups $[P=0.55$; Figure $4 \mathrm{~B}]$. Table 3 shows the causes of death for the two groups. The rituximab group tended to have a higher number of patients with non-recurrence-related death, including one patient who had AMR. 
Table 2. Clinico-pathological and perioperative characteristics of patients between rituximab and control groups

\begin{tabular}{|c|c|c|c|}
\hline Characteristics & Rituximab G $(n=31)$ & Control G $(n=86)$ & $P$ value \\
\hline Age (years) & $60(41-68)$ & $59(29-69)$ & 0.35 \\
\hline Sex (male/female) & $20(65 \%) / 11$ & $61(71 \%) / 25$ & 0.51 \\
\hline Etiology & & & 0.12 \\
\hline Viral hepatitis C & $13(42 \%)$ & $53(62 \%)$ & \\
\hline Viral hepatitis B & $8(26 \%)$ & $21(24 \%)$ & \\
\hline Viral hepatitis C/B & $1(3 \%)$ & $2(2 \%)$ & \\
\hline Others & $9(29 \%)$ & $10(12 \%)$ & \\
\hline $\operatorname{AFP}(n g / m L)$ & $8.8(2.3-28074)$ & $45.3(1.3-19912)$ & 0.07 \\
\hline $\mathrm{DCP}(\mathrm{mAU} / \mathrm{mL})$ & $48(6-3250)$ & $46(6-12523)$ & 0.89 \\
\hline Child-Pugh classification (A/B/C) & $3(10 \%) / 9(29 \%) / 19(61 \%)$ & $10(12 \%) / 36(42 \%) / 40(46 \%)$ & 0.36 \\
\hline MELD & $16(8-35)$ & $15(6-41)$ & 0.64 \\
\hline Maximum tumor size $(\mathrm{cm})$ & $2.3(0.7-4.6)$ & $2.2(0.8-8.8)$ & 0.72 \\
\hline Number of tumors & $2(1-186)$ & $2(1-21)$ & 0.02 \\
\hline Graft type (rt graft/others) & $21(68 \%) / 10$ & $62(72 \%) / 24$ & 0.65 \\
\hline GRWR & $0.81(0.62-1.34)$ & $0.90(0.56-1.54)$ & 0.36 \\
\hline Operation time (min) & $947(703-1288)$ & $784(557-1277)$ & $<0.01$ \\
\hline Blood loss $(\mathrm{mL})$ & $5807(1700-28560)$ & $5915(2300-30418)$ & 0.62 \\
\hline Splenectomy & $17(55 \%)$ & $58(67 \%)$ & 0.28 \\
\hline Classification (well/mod/poor) & $1(3 \%) / 28(90 \%) / 2(7 \%)$ & $13(15 \%) / 55(64 \%) / 18(21 \%)$ & 0.02 \\
\hline Microvascular invasion & $4(13 \%)$ & $23(27 \%)$ & 0.12 \\
\hline Preoperative treatment & $23(74 \%)$ & $52(60 \%)$ & 0.17 \\
\hline \multicolumn{4}{|l|}{$\mathrm{ABO}$ incompatibility } \\
\hline Identical, compatible/incompatible & $1(3 \%) / 30(97 \%)$ & $86(100 \%) / 0(0 \%)$ & $<0.01$ \\
\hline Donor-specific antibody-strong positive & $3(10 \%)$ & $0(0 \%)$ & 0.02 \\
\hline AMR & $4(13 \%)$ & $1(1 \%)$ & 0.02 \\
\hline ATMR & $10(32 \%)$ & $16(19 \%)$ & 0.14 \\
\hline Milan criteria met (met/exceeding) & $25(81 \%) / 6$ & $58(67 \%) / 28$ & 0.17 \\
\hline UCSF criteria met (met/exceeding) & $27(87 \%) / 4$ & $62(72 \%) / 24$ & 0.09 \\
\hline Kyoto criteria met (met/exceeding) & $26(84 \%) / 5$ & $68(81 \%) / 16$ & 0.72 \\
\hline
\end{tabular}

AFP: Alpha-fetoprotein; AMR: antibody-mediated rejection; ATMR: acute T-cell mediated rejection; DCP: des-gamma-carboxy prothrombin; GRWR: graft/recipient body weight ratio; MELD: model for end-stage liver disease; UCSF: University of California San Francisco.

Next, the outcomes of rituximab ( $v$ s. control) administration between the 2 groups were analyzed based on patient selection criteria. In the subgroup analysis as per the MC, the one-, three-, and five-year OS rates in the groups with and without rituximab administration were $84 \%, 79 \%$, and $70 \% v$ s. $88 \%, 86 \%$, and $86 \%$, respectively; survival of the rituximab group tended to be lower than that of the control group $[P=0.07$; Figure $5 \mathrm{~A}]$. On the other hand, one-, three-, and five-year RRs as per MC were $0 \%, 0 \%$, and $6 \% v s .0 \%, 2 \%$, and $2 \%$, with and without rituximab administration respectively; these differences were not significant $[P=$ 0.49; Figure $5 \mathrm{~B}]$. In patients beyond the MC, the one-, three-, and five-year OS rates were $100 \%, 67 \%$, and $67 \%$ vs. $93 \%, 74 \%$, and $74 \%$ with and without rituximab administration respectively; survival rates were not significant between groups $[P=0.78$; Figure $6 \mathrm{~A}]$. Lastly, in patients beyond the MC, the one-, three-, and five-year RRs with and without rituximab administration were $17 \%, 17 \%$, and $17 \%$ vs. $15 \%, 27 \%$, and $31 \%$, respectively; these values were not significant between groups $[P=0.56$; Figure $6 \mathrm{~B}]$.

In patients, as per the UCSF criteria, the one-, three-, and five-year OS rates in the groups with and without rituximab administration were $85 \%, 81 \%$, and $72 \% v s .89 \%, 87 \%$, and $87 \%$, respectively; survival of the rituximab group tended to be lower than that of the control group $[P=0.07$; Figure $7 \mathrm{~A}]$. On the other hand, 
Table 3. Cause of death between rituximab group and control group

\begin{tabular}{lll}
\hline & $\begin{array}{l}\text { Rituximab G } \\
(\boldsymbol{n}=\mathbf{3 1})\end{array}$ & $\begin{array}{l}\text { Control G } \\
(\boldsymbol{n = 8 6 )}\end{array}$ \\
\hline Cause & 2 & 7 \\
Recurrence & 9 & 0.76 \\
Non-recurrence & 4 & 12 \\
Infection & 2 & 7 \\
$\quad$ Graft failure & 3 & 3 \\
\multicolumn{1}{c}{ Others } & AMR (1), brain bleeding (1), & 2 \\
& PV thrombosis(1) & HCV recurrence (1), Unknown (1) \\
Total number of death & 11 & 19 \\
\hline
\end{tabular}

AMR: antibody-mediated rejection; HCV: hepatitis C virus; PV: portal vein.

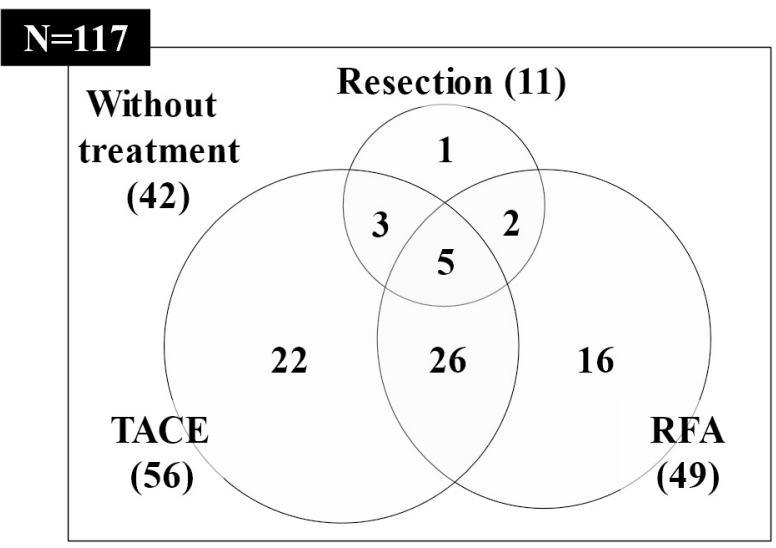

Figure 2. Preoperative treatment for HCC. HCC: Hepatocellular carcinoma; TACE: transcatheter arterial chemoembolization; RFA: radiofrequency ablation.

A

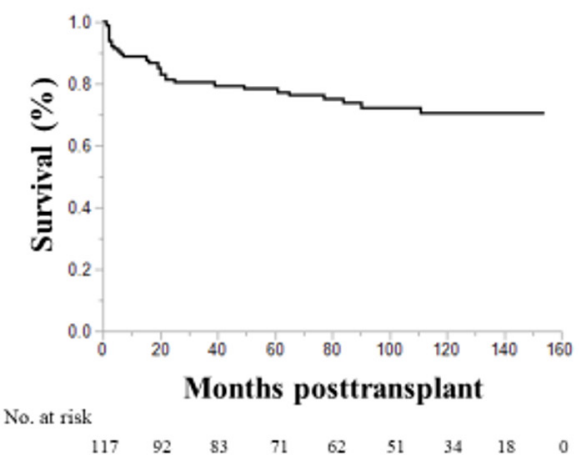

B

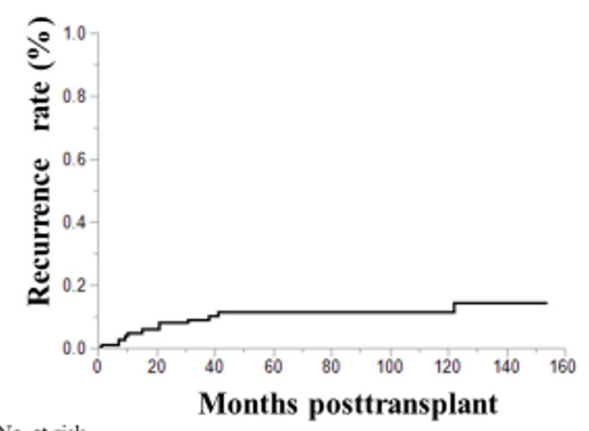

No. at risk
Months posttransplant

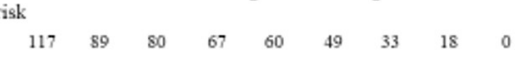

Figure 3. Overall survival (A) and recurrence (B) rates after LT in patients with HCC. HCC: Hepatocellular carcinoma; LT: liver transplantation.

the one-, three-, and five-year RRs as per UCSF with and without rituximab administration were $0 \%, 0 \%$, and $5 \% v s .0 \%, 2 \%$, and $2 \%$, respectively, and were not significant $[P=0.50$; Figure $7 \mathrm{~B}]$. In patients beyond 
A

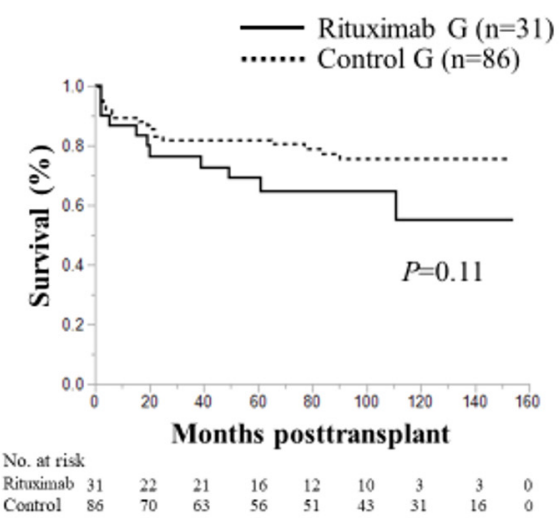

B

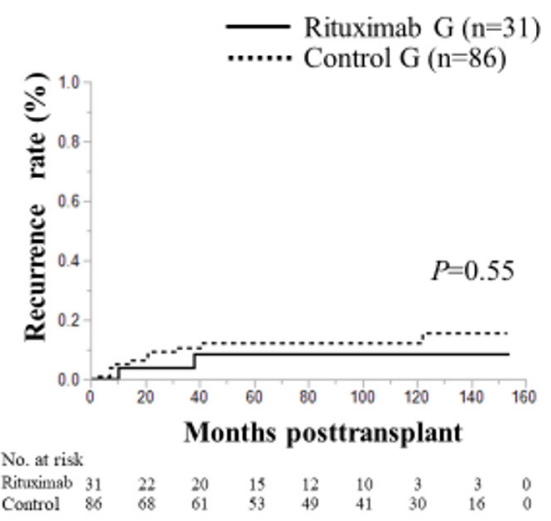

Figure 4. Overall survival $(A)$ and recurrence $(B)$ rates following $L T$ in reference to rituximab administration in patients with HCC. HCC: Hepatocellular carcinoma; MC: Milan criteria; LT: liver transplantation.

A

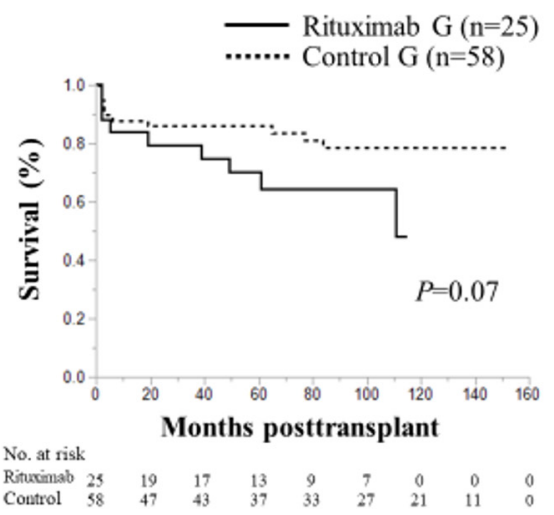

B

Within Milan

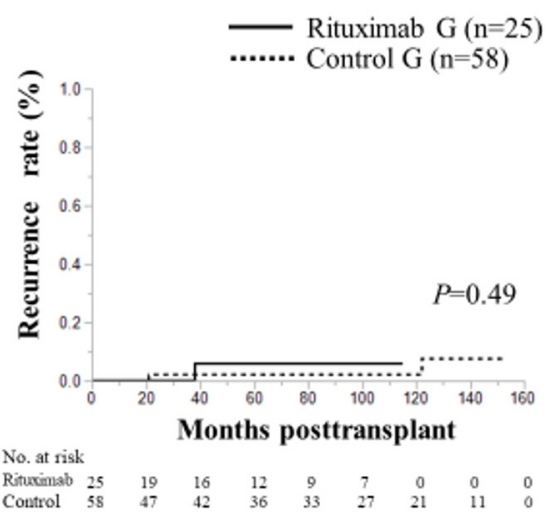

Figure 5. Overall survival $(A)$ and recurrence $(B)$ rates following LT in reference to rituximab administration in patients with $\mathrm{HCC}$ within the MC. HCC: Hepatocellular carcinoma; MC: Milan criteria; LT: liver transplantation.

the UCSF, the one-, three-, and five-year OS rates with and without rituximab administration were $100 \%$, $50 \%$, and $50 \%$ vs. $91 \%, 70 \%$, and $70 \%$, respectively $[P=0.48$; Figure $8 \mathrm{~A}]$; while the. RRs were $25 \%, 25 \%$, and $25 \%$ vs. $18 \%, 32 \%$, and $36 \%$, respectively for the corresponding years between the 2 groups $[P=0.78$; Figure $8 \mathrm{~B}]$. One patient within the UCSF in the rituximab group had a recurrence at 38 months after LT. The pathological grade of tumor was poorly differentiated, while 2 patients within the UCSF in the control group had recurrences at 21 and 122 months after LT. The pathological grade of the tumors was moderately differentiated. On the other hand, 1 patient beyond the UC in the rituximab group had recurrence of moderately differentiated tumor at 10 months after LT, and eight patients beyond the UC in the control group had recurrence after LT. The median period of recurrence was 12 months (range; 1-41 months) and the pathological grade of tumor in these patients was moderately differentiated for 2 patients and poorly differentiated for 6 patients.

In the patients as per the KC criteria, the one-, three-, and five-year OS rates in groups with and without rituximab administration were $88 \%, 76 \%$, and $67 \%$ s. $93 \%, 88 \%$, and $88 \%$, respectively, and the survival rate was significantly lower in the rituximab group than in the control group $[P=0.014$; Figure $9 \mathrm{~A}]$; 
A

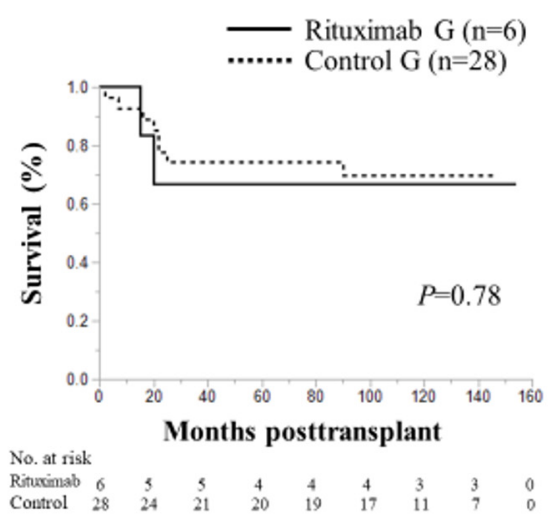

B Beyond Milan

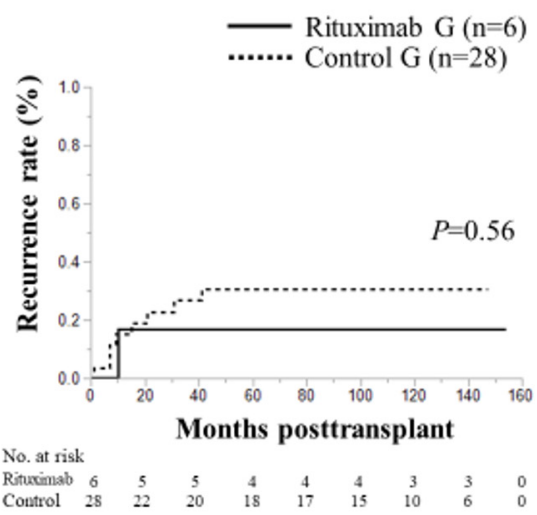

Figure 6. Overall survival (A) and recurrence (B) rates following LT according to rituximab administration in patients with HCC beyond the MC. HCC: Hepatocellular carcinoma; MC: Milan criteria; LT: liver transplantation.

$\mathbf{A}$

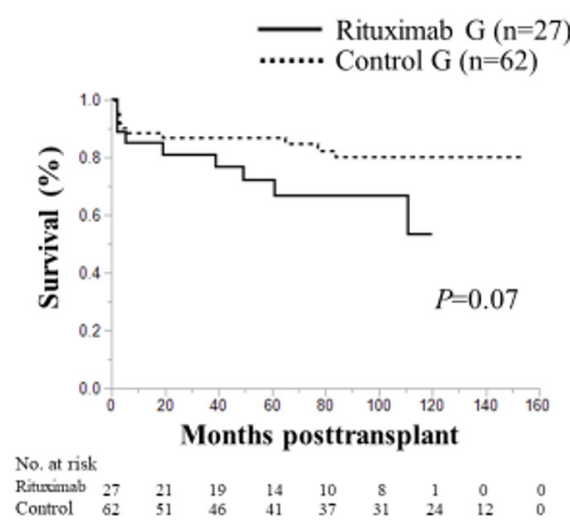

B Within UCSF

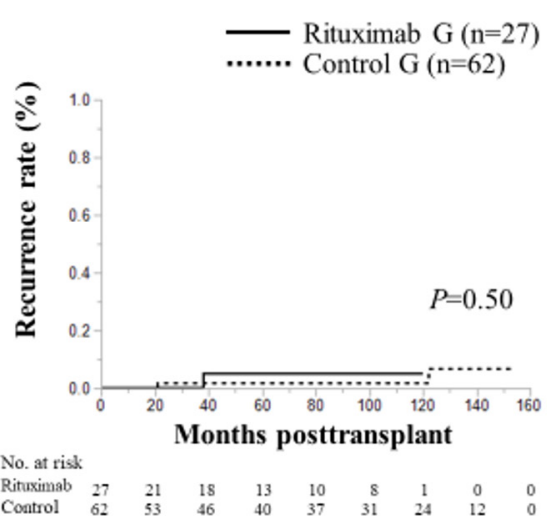

Figure 7. Overall survival (A) and recurrence (B) rates following LT in relation to rituximab administration in patients with HCC as per UCSF critera. HCC: Hepatocellular carcinoma; LT: liver transplantation; UC: UCSF criteria.

corresponding RRs as per KC with and without rituximab administration were $5 \%, 5 \%$, and $10 \% v$ s. $2 \%, 7 \%$, and $7 \%$ for one-, three-, and five-years respectively, and showed nosignificance between groups $[P=0.67$; Figure $9 \mathrm{~B}]$. In patients beyond the $\mathrm{KC}$, the one-, three-, and five-year OS rates in the groups with and without rituximab administration were $80 \%, 80 \%$, and $80 \%$ vs. $75 \%, 63 \%$, and $63 \%$, respectively, and the survival rates did not differ significantly between the 2 groups $[P=0.56$; Figure $10 \mathrm{~A}]$; similar, the one-, three-, and five-year RRs in the groups with and without rituximab administration were $0 \%, 0 \%$, and $0 \% v s$. $14 \%, 22 \%$, and $31 \%$, respectively, without significance $[P=0.19$; Figure $10 \mathrm{~B}] .2$ patients within the KC in the rituximab group had recurrence at 10 and 38 months after LT; the grade of tumor biology was moderately and poorly differentiated, respectively. Moreover, 4 patients within the KC in the control group had recurrences at a median period of 18 months (range; 9-31 months) after LT. The grade of tumor biology was moderately for 1 patient and poorly differentiated for 3 patients. There were 5 patients beyond the KC in the control group who had recurrence after LT and there was no patients beyond the KC in the rituximab group. Median period of recurrence was 21 months (range; 1-122 months). The grade of tumor biology in these patients was moderately for 3 patients and poorly differentiated for 2 patients. 
A

Beyond UCSF

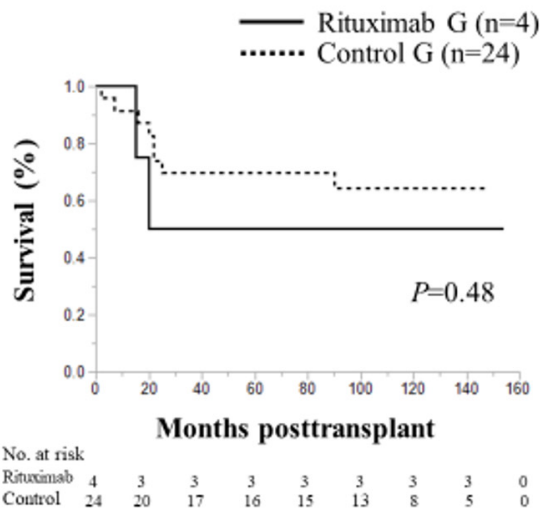

B Beyond UCSF

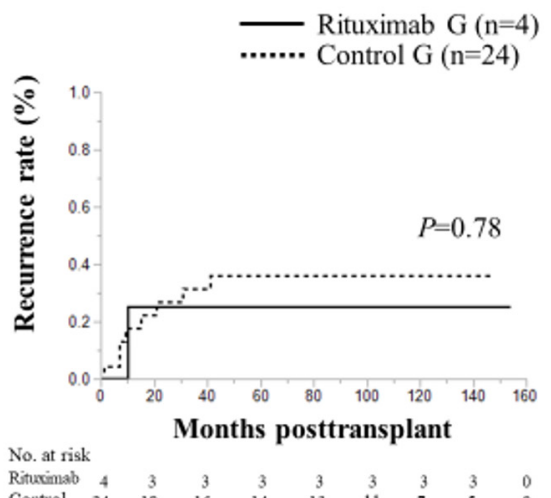

Figure 8. Overall survival (A) and recurrence (B) rates following LT according to rituximab administration in patients with HCC beyond the UCSF. HCC: Hepatocellular carcinoma; LT: liver transplantation; UC: UCSF criteria.

A

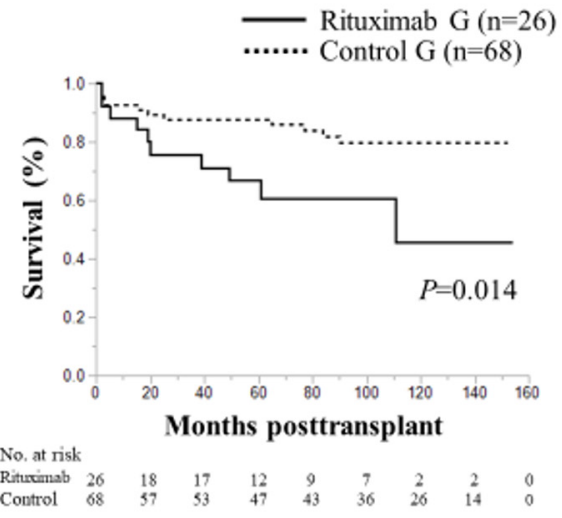

B Within Kyoto

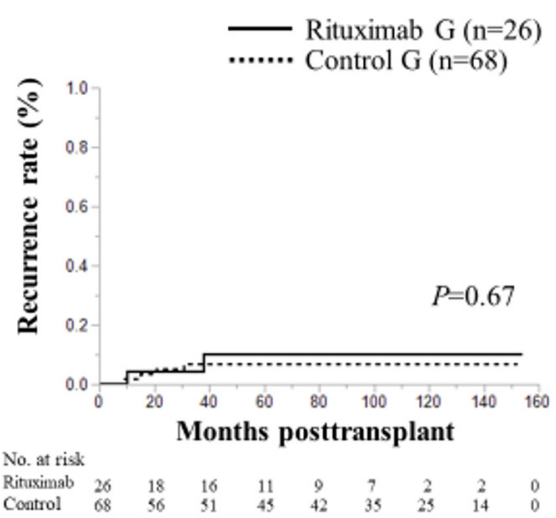

Figure 9. Overall survival (A) and recurrence (B) rates following LT according to rituximab administration in patients with HCC within the KC. HCC: Hepatocellular carcinoma; KC: Kyoto criteria; LT: liver transplantation.

Finally, we analyzed risk factors associated with HCC recurrence while focusing on pretransplant factors [Table 4]. Univariate analysis identified the AFP level at transplant, maximum tumor size, and preoperative treatment as significant risk factors for HCC recurrence after LT $(P=0.003,<0.001$, and 0.015 , respectively). On multivariate analysis, maximum tumor size $(P=0.003)$ and preoperative treatment $(P=0.024)$ were identified as independent risk factors for HCC recurrence. However, rituximab administration was not an independent risk factor for recurrence.

\section{DISCUSSION}

This retrospective analysis provides evidence for the long-term outcomes of LDLT for HCC in patients with and without rituximab-based prophylaxis. This is the first analysis that shows the effects of rituximab administration on HCC recurrence in patients who underwent DSA-positive LDLT, as well as ABO-I transplant. The RR in patients who were administered rituximab before LDLT was not significantly different from that in patients who did not receive rituximab. Therefore, LDLT that requires rituximab desensitization therapy is considered a safe and feasible treatment for HCC. 
Table 4. Univariate and multivariate analysis of risk factors for $\mathrm{HCC}$ recurrence

\begin{tabular}{|c|c|c|c|c|c|c|}
\hline \multirow{2}{*}{ Variable } & \multicolumn{3}{|c|}{ Univariate } & \multicolumn{3}{|c|}{ Multivariate } \\
\hline & HR & $95 \% \mathrm{Cl}$ & $P$ & HR & $95 \% \mathrm{Cl}$ & $\mathbf{P}$ \\
\hline Age & 0.936 & $0.866-1.019$ & 0.122 & & & \\
\hline Sex (male) & 4.769 & $0.927-87.155$ & 0.064 & & & \\
\hline Rituximab administration & 0.631 & $0.097-2.423$ & 0.535 & 0.899 & $0.133-3.780$ & 0.894 \\
\hline MELD score & 0.888 & $0.771-1.005$ & 0.061 & & & \\
\hline $\log _{10}$ AFP & 2.480 & $1.373-4.423$ & 0.003 & 1.351 & $0.741-2.471$ & 0.320 \\
\hline $\log _{10} \mathrm{DCP}$ & 1.919 & $1.004-3.485$ & 0.049 & 1.577 & $0.739-3.241$ & 0.228 \\
\hline Maximum tumor size & 2.093 & $1.561-2.809$ & $<0.001$ & 1.777 & $1.226-2.559$ & 0.003 \\
\hline Number of tumors & 1.003 & $0.960-1.017$ & 0.790 & & & \\
\hline Preoperative treatment & 7.111 & $1.382-129.960$ & 0.015 & 6.706 & $1.242-124.215$ & 0.024 \\
\hline
\end{tabular}

AFP: Alpha-fetoprotein; Cl: confidence interval; DCP: des-gamma-carboxy prothrombin; HCC: hepatocellular carcinoma; HR: hazard ratio; MELD: model for end-stage liver disease.

$\mathbf{A}$

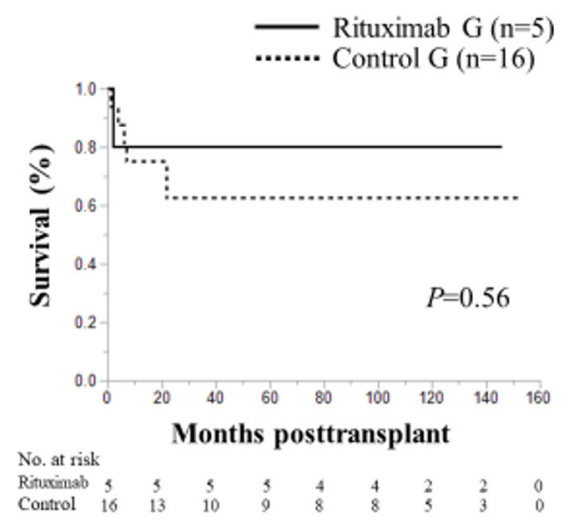

B

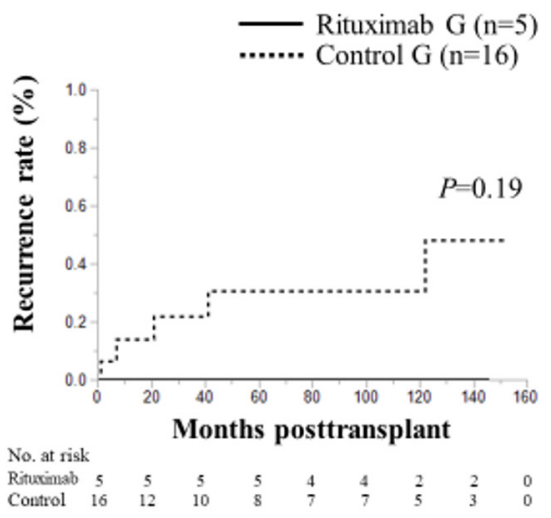

Figure 10. Overall survival (A) and recurrence (B) rates following LT according to rituximab administration in patients with HCC beyond the KC. HCC: Hepatocellular carcinoma; KC: Kyoto criteria; LT: liver transplantation.

ABO-I transplants have increased since rituximab first appeared in $2004^{[20]}$. Egawa et al ${ }^{[20]}$ reported that OS for HCC was significantly worse in ABO-I transplants than in ABO-C transplants. Moreover, in cases of ABO-I transplants for HCC, OS was significantly better in the group with rituximab administration than in the group without rituximab administration. In the present study, since 2006, there were no significant differences in the survival rates between the control group and the rituximab group that consisted of patients with ABO-I and who were DSA-positive. However, in the subgroup analysis based on selection criteria, the survival rate of the rituximab group in patients within the MC, UCSF or KC was worse than that of the control group. Others ${ }^{[8-10]}$ have similarly examined cases within the MC and shown no significant differences in $\mathrm{OS}$ and RR by $\mathrm{ABO}$ incompatibility.

There are some possible reasons for the differences seen in OS in the present study. The first is an issue with AMR. 4 out of 31 patients in our study had AMR, and one of them died from AMR. In fact, $25 \%$ of ABO-I patients with and without HCC in our hospital had AMR, and $40 \%$ of them died from AMR (data not shown). This high occurrence rate of AMR might be influenced by the initial dose (300 $\mathrm{mg}$ ) and/or timing of rituximab administration; some patients with acute liver failure received rituximab within 1 week before LT. Furthermore, the threshold for PE or intravenous immunoglobulin titers in patients might be different. 
Another reason might be the study period because patients with rituximab administration also had a higher frequency of steroid-use infections. In addition to improvements in surgical techniques and perioperative management, including changes in IS protocol, remarkable advances in diagnostic imaging have helped facilitate the discovery of tumors while they are relatively small in size and number before they progress to more critical situations. Therefore, it seems that there was little difference in the survival rate in recent years. Regarding the recurrence rates, there were no significant differences between the rituximab and the control groups in patients within or beyond each selection criteria. In fact, rituximab administration was not an independent risk factor for recurrence. Maximum tumor size and preoperative treatment were identified as independent risk factors for HCC recurrence in this study. Since 2007, we excluded HCC patients who were beyond $\mathrm{KC}$ from transplant indication, in addition to those within MC who had DCP concentration $>400 \mathrm{mAU} / \mathrm{mL}$. This selection might have affected results of multivariate analysis in this study. However, it is difficult to ascertain whether rituximab itself affects the recurrence of HCC or not because of its single-dose administration in most cases. Effects of other IS or biological malignancy of the tumor itself might also be involved. Indeed, there were no well-differentiated tumors in patients with recurrence in this study.

Rituximab works on the $\mathrm{CD} 20$ antigen present on B cells and reduces the production of $\mathrm{B}$ cells that are mainly responsible for $\mathrm{AMR}^{[18]}$. B cells in the body are temporarily depleted, and antibody production is suppressed. Administration of steroids, on the other hand, reduces T cells, especially CD4-positive helper T cells ${ }^{[21]}$. Since lymphocytic infiltration of the tumor and a high $\mathrm{CD} 4^{+}: \mathrm{CD}^{+} \mathrm{T}$-cell ratio are associated with a reduced risk of tumor recurrence after $\mathrm{LT}$ for $\mathrm{HCC}^{[22]}$, more steroids might increase the risk of HCC recurrence ${ }^{[23,24]}$. In the present study, TAC and MMF were administered to both groups in similar ways, and there were no significant differences in RR between the rituximab group and the control group within the MC, UCSF, or KC. Since the use of postoperative steroids after the introduction of rituximab has been standardized, we avoided steroid overdose, and this might be the reason why there was no significant difference between the rituximab group and the control group in the RR of HCC.

In the case of adult LDLT, the presence of DSA-positive donors was as problematic as ABO-I LT. It is difficult to avoid DSA-positive donors due to the current situation in Japan, where deceased donor LT is rarely performed compared with Western countries. Since organ donation is from a close relative donor in LDLT, there is sometimes a common HLA even if there are fewer donor candidates ${ }^{[25]}$. Moreover, adult women are often sensitized to HLA through pregnancy, and there are cases in which LT from DSA-positive donors is unavoidable. Therefore, it is essential to pay attention to AMR and manage the perioperative period. In our institute, measuring DSA levels started in 2009. In the present study, there were 3 such patients, and 1 of the 3 patients died of infection. The other two are still alive without HCC recurrence. Since the number of DSA-positive HCC patients has been small, experience with more cases is needed in the future.

Considering the type of recurrence, extrahepatic recurrence $(n=9)$ was more frequent than intrahepatic $(n$ = 3) (data not shown). Especially within KC, 6 of 94 patients had 3 intrahepatic and 3 extrahepatic recurrences. On the other hand, 5 of 21 patients beyond the KC had 5 extrahepatic recurrences. The immune-checkpoint inhibitors, replacing the role of tyrosine kinase inhibitors, might be appropriate for such cases although data from adequate patient cases must be accumulated to confirm the effect. However, it is unclear how different immunosuppressive regimens affect the recurrence of HCC. Therefore, it is crucial to apply these criteria to lower recurrence rates of HCC. 
Some limitations must be considered when interpreting the results of this study. First, this study was retrospective in design and selection biases could have affected the analysis. Second, the number of targeted patients for this study was relatively small. Third, this study was performed at a single center. A larger cohort study is therefore needed to confirm the present findings.

In conclusion, retrospective analysis of data in this study shows that rituximab administration does not affect HCC recurrence after LDLT.

\section{DECLARATIONS}

\section{Authors' contributions}

Made contributions to conception and design of the study, performed data acquisition, data analysis and interpretation and manuscript writing: Kamo N

Made contributions to conception and design of the study, performed technical and material support: Yagi S

\section{Availability of data and materials}

Not applicable.

\section{Financial support and sponsorship}

None.

\section{Conflicts of interest}

Both authors declared that there are no conflicts of interest.

\section{Ethical approval and consent to participate}

This study was approved by the ethics committee of Kyoto University (approval no. R1473-3).

\section{Consent for publication}

Not applicable.

\section{Copyright}

(c) The Author(s) 2021.

\section{REFERENCES}

1. Bray F, Ferlay J, Soerjomataram I, Siegel RL, Torre LA, Jemal A. Global cancer statistics 2018: GLOBOCAN estimates of incidence and mortality worldwide for 36 cancers in 185 countries. CA Cancer J Clin 2018;68:394-424. DOI PubMed

2. Chen CL, Fan ST, Lee SG, Makuuchi M, Tanaka K. Living-donor liver transplantation: 12 years of experience in Asia. Transplantation 2003;75:S6-11. DOI PubMed

3. Yoon YI, Lee SG. Living donor liver transplantation for hepatocellular carcinoma: An Asian Perspective. Dig Dis Sci 2019;64:9931000. DOI PubMed

4. Hong G, Yi NJ, Suh SW, et al. Preoperative selective desensitization of liver donor liver transplant recipients considering the degree of T lymphocyte cross-match titer, model for end-stage liver disease score, and graft liver volume. J Korean Med Sci 2014;29:640-7. DOI PubMed PMC

5. Egawa H. Challenge to ABO blood type barrier in living donor liver transplantation. Hepatobiliary Pancreatic Dis Int 2020;19:342-8. DOI PubMed

6. Wesson RN, Etchill EW, Garonzik-Wang J. Application and interpretation of histocompatibility data in liver transplantation. Curr Opin Organ Transplant 2017;22:499-504. DOI PubMed

7. Egawa H, Teramukai S, Haga H, et al. Impact of rituximab desensitization on blood-type-incompatible adult living donor liver transplantation: a Japanese multicenter study. Am J Transplant 2014;14:102-14. DOI PubMed

8. Kim SH, Lee EC, Na BG, Park SJ. Impact of ABO-incompatibility on hepatocellular carcinoma recurrence after living donor liver transplantation. Eur J Surg Oncol 2019;45:180-6. DOI PubMed

9. Kim JM, Kwon CHD, Joh JW, et al. ABO-incompatible living donor liver transplantation with rituximab and total plasma exchange does not increase hepatocellular carcinoma recurrence. Transplantation 2018;102:1695-701. DOI PubMed 
10. Yoon YI, Song GW, Lee SG, et al. Outcome of ABO-incompatible adult living-donor liver transplantation for patients with hepatocellular carcinoma. J Hepatol 2018;68:1153-62. DOI PubMed

11. Kamo N, Kaido T, Hammad A, et al. Impact of elderly donors for liver transplantation: a single-center experience. Liver transpl 2015;21:591-8. DOI PubMed

12. Kamo N, Kaido T, Hamaguchi Y, et al. Impact of enteral Nutrition with an immunomodulating diet enriched with hydrolyzed whey peptide on infection after liver transplantation. World J Surg 2018;42:3715-25. DOI PubMed

13. Ueda Y, Uemoto S. Interferon-free therapy for hepatitis C in liver transplantation recipients. Transplantation 2016;100:54-60. DOI PubMed

14. Ueda Y, Marusawa H, Kaido T, et al. Efficacy and safety of prophylaxis with entecavir and hepatitis B immunoglobulin in preventing hepatitis B recurrence after living-donor liver transplantation. Hepatol Res 2013;43:67-71. DOI PubMed

15. Inomata $\mathrm{Y}$, Tanaka K, Egawa H, et al. The evolution of immunosuppression with FK 506 in pediatric living related liver transplantation. Transplantation 1996;61:247-52. DOI PubMed

16. Takada Y, Ueda M, Ito T, et al. Living donor liver transplantation as a second-line therapeutic strategy for patients with hepatocellular carcinoma. Liver Transpl 2006;12:912-9. DOI PubMed

17. Fukumitsu K, Hammad A, Kaido T, et al. Validation of steroid-free immunosuppression regimen after liver transplantation. $J$ Clin Gastroensterol Treat 2015;1:1-4.

18. Egawa H, Umeshita K, Uemoto S. Optimal dosage regimen for rituximab in ABO-incompatible living donor liver transplantation. $J$ Hepatobiliary Pancreat Sci 2017;24:89-94. DOI PubMed

19. Takada Y, Ito T, Ueda M, et al. Living donor liver transplantation for patients with HCC exceeding the Milan criteria: a proposal of expanded criteria. Dig Dis 2007;25:299-302. DOI PubMed

20. Egawa H, Shimamura T, Todo S. Impact of desensitization for ABO-blood bar- rier on living donor liver transplantation for hepatocellular carcinoma. Nihon Shokakibyo Gakkai Zasshi 2014;111:892-8.

21. Olnes MJ, Kotliarov Y, Biancotto A, et al. Effects of systemically administered hydrocortisone on the human immunome. Sci Rep 2016;14:23002. DOI PubMed PMC

22. Unitt E, Marshall A, Gelson W, et al. Tumor lymphocytic infiltrate and recurrence of hepatocellular carcinoma following liver transplantation. J Hepatol 2006;45:246-253. DOI PubMed

23. Lee JY, Kim YH, Yi NJ, et al. Impact of immunosuppressant therapy on early recurrence of hepatocellular carcinoma after liver transplantation. Cli Mol Hepatol 2014;20:192-203. DOI PubMed PMC

24. Wu LW, Tai Q, Zhu XF, He XS. Safety and efficacy of four steroid-minimization protocols in liver transplant recipients: 3-year follow-up in a single center. J Dig Dis 2013;14:38-44.

25. Yoshizawa A. The impact of donor-specific anti-HLA antibodies on liver transplantation. $M H C$ 2017;24:134-42. DOI 